\title{
ECDC definitions and methods for the surveillance of healthcare-associated infections in intensive care units
}

\author{
Diamantis Plachouras ${ }^{1 *} \mathbb{D}$, Alain Lepape ${ }^{2,3}$ and Carl Suetens ${ }^{1}$
}

๑) 2018 The Author(s), corrected publication September/2018

On 5 May 2017, the European Centre for Disease Prevention and Control (ECDC) published its updated protocol for surveillance of healthcare-associated infections (HAIs) and prevention indicators in European intensive care units (ICUs) [1]. The ECDC's Healthcare-Associated Infections surveillance Network (HAI-Net) has been coordinating European surveillance of HAIs in ICUs since 2008 with participation of 15 countries and 1365 ICUs in 2015 [2]. The methodology was originally developed by the Hospitals in Europe Link for Infection Control through Surveillance (HELICS) project in 2000-2002 [3] and adopted by ECDC in 2010. A revision of the first protocol was agreed at consensus expert meetings in 2013-2015.

The objective of the ECDC surveillance protocol is the standardisation of case definitions, data collection and reporting procedures for hospitals participating in national or regional surveillance of HAIs in ICUs across Europe in order to facilitate the collection of comparable epidemiological data as a basis for interventions and the communication and exchange of experience of national surveillance systems. The case definitions of HAIs aim to accommodate differences, across Europe, in diagnostic practices such as taking microbiological samples for the diagnosis of HAIs. The minimal requirement for participation in surveillance of HAIs in ICUs is surveillance of bloodstream infections (BSIs) and pneumonia (PN).

\footnotetext{
*Correspondence: diamantis.plachouras@ecdc.europa.eu

${ }^{1}$ European Centre for Disease Prevention and Control, Gustav III:S boulevard 40, 16973 Solna, Sweden

Full author information is available at the end of the article

The original version of this article was revised due to a retrospective
} Open Access order.
Reporting of urinary tract infections (UTIs) and catheter-related infections (CRIs) is optional. The data are collected by the national surveillance systems and submitted annually to ECDC. The results are published on the ECDC website [2]. A software application, HelicsWin. Net, is available free-of-charge for entry of HAI surveillance data (https://ecdc.europa.eu/en/publications-data/ helicswinnet-hwn).

The latest update of the ECDC protocol was triggered by the unexplained, large variation in the prevalence of HAIs among European countries reported by the ECDC point prevalence survey of HAIs and antimicrobial use in European acute care hospitals 2011-2012. Structure and process indicators for infection prevention and control and antimicrobial stewardship (Table 1), as well as a new variable on the outcome of the HAI were included in the latest version of the surveillance protocol. These modifications were developed by ECDC and HAI-Net ICU experts and agreed during an HAI-Net ICU meeting in February 2015.

The ECDC case definitions applied for surveillance of HAIs in European ICUs are presented in Table 1a in the supplementary material.

The case definition for BSI addresses both primary and secondary BSIs. A definition for CRI establishes the microbiological criteria for assignment of the catheter as the origin of the BSI. When CRIs are not specifically reported, BSIs for which the same microorganism was cultured from the catheter or when symptoms improved within $48 \mathrm{~h}$ after removal of the catheter are reported as BSIs with origin central venous catheter (CVC). In this way, it is possible to assess the burden of BSIs linked to catheters in ICUs with different local practices for microbiological confirmation through culture of the catheter

\section{Springer}


Table 1 Structure and process indicators for infection prevention control and antimicrobial stewardship in intensive care units (ICUs)

\begin{tabular}{lll} 
Area & Indicator & Metric \\
\hline Hand hygiene & Alcohol hand rub consumption in ICU during the previous year & Litres per 1000 patient-days \\
Staffing & Registered nurse hours in ICU & Hours per 1000 patient-days \\
& Nursing assistant hours in ICU & Hours per 1000 patient-days \\
Antimicrobial stewardship & Review antimicrobial therapy within $72 \mathrm{~h}$ & Proportion (\%) compliant observations \\
Intubation & Endotracheal pressure controlled and/or corrected at least twice a day & Proportion (\%) compliant observations \\
& Oral decontamination using oral antiseptics at least twice a day & Proportion (\%) compliant observations \\
& Position of the patient not supine & Proportion (\%) compliant observations \\
Central venous catheter & Catheter site dressing is not damp, loose or visibly soiled & Proportion (\%) compliant observations \\
\hline
\end{tabular}

tip or taking blood samples through the catheter for quantitative culture or differential time to positivity. This definition differs from the definition of the National Healthcare Safety Network of the US Centers for Disease Control and Prevention (CDC/NHSN) of central line-associated bloodstream infection (CLA-BSI) that includes all primary BSIs with a central line in place for at least 2 days on the date of the BSI [4]. However, it is possible to calculate CLA-BSI rates with HAI-Net ICU data to perform comparisons with data from the US and other surveillance networks that use this definition. The definitions for CRI and BSI with origin CVC were preferred, as they avoid incriminating the catheter for BSI cases with unidentified origin. Such cases may be the result of translocation or a difficult to confirm source and linking them to a central vascular catheter may lead to overestimation of the incidence and dilute the effect of preventive interventions [5]. The CRI definition has been applied in studies that assess the effect of prevention efforts [6].

For pneumonia, the ECDC case definition (based on the 2002 NHSN definition) applies imaging, clinical and microbiological criteria and consists of five levels (PN1PN5), from PN1 requiring microbiological confirmation from minimally contaminated specimens to PN5 requiring no positive microbiological data. These levels address differences in diagnostic practices across Europe and intend to ensure comprehensiveness and inform the interpretation of the results. The definitions primarily aim to support infection prevention and control but they also encourage microbiological confirmation that may impact clinical management in terms of treatment decisions and especially antimicrobial use. The definition for ventilator-associated pneumonia (VAP) differs from the current CDC/NHSN definition of ventilatorassociated events (VAE), which was implemented in the USA in 2013 [5]. HAI-Net maintained the previous definition for pneumonia and VAP, as it was considered more relevant from both clinical and prevention perspectives.
The definition is in line with the one used in prevention studies and diagnostic and treatment guidelines and has been used in studies that demonstrate the effect of preventive interventions [7]. Furthermore, a recent metaanalysis indicated that VAE surveillance does not detect cases of VAP accurately [8]. A comparison with the current CDC/NHSN definition of VAE and probable VAP [4] showed discordance for VAP, with agreement in only four of 61 cases identified as VAP by either one or the other definition [9]. In contrast, a study comparing the ECDC definition with the previous US definition for VAP had demonstrated very high concordance when applying both definitions in the same group of patients [10].

The updated ECDC protocol for surveillance of HAIs in European ICUs combines outcome indicators (incidence of HAIs, microbiology and outcome) with structure and process indicators for infection prevention and control and antimicrobial stewardship. It aims to provide information that is useful and actionable at hospital, regional and national level both for prevention of HAIs and policymaking. EU/EEA countries are invited to apply the protocol in national surveillance systems and participate in European surveillance fostering an EU-wide collaboration to support the prevention of HAIs.

\section{Electronic supplementary material}

The online version of this article (https://doi.org/10.1007/s00134-018-5113-0) contains supplementary material, which is available to authorized users.

\section{Author details \\ ${ }^{1}$ European Centre for Disease Prevention and Control, Gustav III:s boulevard 40, 16973 Solna, Sweden. ${ }^{2}$ Clinical Research Unit, Critical Care, Lyon Sud Uni- versity Hospital, Hospices Civils de Lyon, Lyon, France. ${ }^{3}$ Laboratory of Emerg- ing Pathogens, International Center for Infectiology Research (CIRI), Inserm U1111 CNRS, UMR5308, ENS de Lyon, UCBL1, Lyon, France.}

\section{Acknowledgements}

Anne Savey, Coordination Center for Healthcare-associated Infection Prevention and Control in South-Eastern France, Lyon, France; Mercedes Palomar, Institut Recerca Biomedica Lleida (IRBLL), Lleida, Spain; Antonella Agodi, University of Catania, Italy; Michael Hismayr, Medical University Vienna, Austria; Karl Mertens, Scientific Institute of Public Health (WIV-ISP), Brussels, 
Belgium; Jana Prattingerová, Regional Public Health Authority, Liberec, Czech Republic; Pille Märtin, West Tallinn Central Hospital, Estonia; Petra Gastmeier, National Reference Center for Surveillance of Nosocomial Infections, Berlin, Germany; Andrea Kurcz, Ministry of Human Capacities, Budapest, Hungary; Maria Luisa Moro, Agenzia Sanitaria e Sociale Regione Emilia-Romagna, Bologna, Italy; Jolanta Asembergiene, National Cancer Institute, Vilnius, Lithuania; Jean-Claude Schmit, Health Directorate, Luxembourg; Elizabeth Anne Scicluna, Infection Control Unit, Mater Dei Hospital, Msida, Malta; Paulo André Fernandes, Program for Prevention and Control of Infections and Resistance to Antimicrobials (PPCIRA), Lisbon, Portugal; Roxana Serban, Regional Centre of Public Health, Bucharest, Romania; Maria Štefkovičová, Alexander Dubcek University, Trenčín, Slovakia; Jodie McCoubrey, Health Protection Scotland, Glasgow, UK; Dominique L. Monnet, European Centre for Disease Prevention and Control, Stockholm, Sweden

\section{Compliance with ethical standards}

\section{Conflicts of interest}

The authors declare that they have no conflict of interest.

\section{Open Access}

This article is distributed under the terms of the Creative Commons Attribution-NonCommercial 4.0 International License (http://creativecommons.org/ licenses/by-nc/4.0/), which permits any noncommercial use, distribution, and reproduction in any medium, provided you give appropriate credit to the original author(s) and the source, provide a link to the Creative Commons license, and indicate if changes were made.

Received: 24 January 2018 Accepted: 21 February 2018 Published online: 24 May 2018

\section{References}

1. European Centre for Disease Prevention and Control (2017) Surveillance of healthcare-associated infections and prevention indicators in European intensive care units. ECDC, Stockholm. https://ecdc.europa.eu/ sites/portal/files/documents/HAl-Net-ICU-protocol-v2.2_0.pdf. Accessed 12 Feb 2018

2. European Centre for Disease Prevention and Control (2016) Healthcareassociated infections acquired in intensive care units. ECDC, Stockholm. https://ecdc.europa.eu/sites/portal/files/documents/AER-HCAI_ICU.pdf. Accessed 12 Feb 2018
3. Suetens C, Savey A, Labeeuw J, Morales I, HELICS-ICU (2002) The ICUHELICS programme: towards European surveillance of hospital-acquired infections in intensive care units. Euro Surveill 7:127-128

4. Centers for Disease Control and Prevention (2018) National Healthcare Safety Network (NHSN) Patient Safety Component Manual. CDC, Atlanta. https://www.cdc.gov/nhsn/pdfs/pscmanual/pcsmanual_current.pdf. Accessed 12 Feb 2018

5. Sihler KC, Chenoweth C, Zalewski C, Wahl W, Hyzy R, Napolitano LM (2010) Catheter-related vs. catheter-associated blood stream infections in the intensive care unit: incidence, microbiology, and implications. Surg Infect (Larchmt) 11:529-534. https://doi.org/10.1089/sur.2009.084

6. van der Kooi T, Sax H, Pittet D, van Dissel J, van Benthem B, Walder B, Cartier V, Clack L, de Greeff S, Wolkewitz M, Hieke S, Boshuizen H, van de Kassteele J, Van den Abeele A, Boo TW, Diab-Elschahawi M, Dumpis U, Ghita C, FitzGerald S, Lejko T, Leleu K, Martinez MP, Paniara O, Patyi M, Schab P, Raglio A, Szilagyi E, Zietkiewicz M, Wu AW, Grundmann H, Zingg W, PROHIBIT consortium (2018) Prevention of hospital infections by intervention and training (PROHIBIT): results of a pan-European cluster-randomized multicentre study to reduce central venous catheterrelated bloodstream infections. Intensive Care Med 44:48-60. https://doi. org/10.1007/s00134-017-5007-6

7. Alvarez-Lerma F, Palomar-Martinez M, Sanchez-Garcia M, Martinez-Alonso M, Alvarez-Rodriguez J, Lorente L, Arias-Rivera S, Garcia R, Gordo F, Anon JM, Jam-Gatell R, Vazquez-Calatayud M, Agra Y (2018) Prevention of ventilator-associated pneumonia: the multimodal approach of the Spanish ICU "Pneumonia Zero" Program. Crit Care Med 46:181-188. https://doi. org/10.1097/CCM.0000000000002736

8. Fan Y, Gao F, Wu Y, Zhang J, Zhu M, Xiong L (2016) Does ventilatorassociated event surveillance detect ventilator-associated pneumonia in intensive care units? A systematic review and meta-analysis. Crit Care 20:338. https://doi.org/10.1186/s13054-016-1506-z

9. Craven TH, Wojcik G, McCoubrey J, Brooks O, Grant E, Reilly J, Laurenson IF, Kefala K, Walsh TS (2018) Lack of concordance between ECDC and CDC systems for surveillance of ventilator associated pneumonia. Intensive Care Med 44:265-266. https://doi.org/10.1007/s00134-017-4993-8

10. Hansen S, Sohr D, Geffers C, Astagneau P, Blacky A, Koller W, Morales I, Moro ML, Palomar M, Szilagyi E, Suetens C, Gastmeier P (2012) Concordance between European and US case definitions of healthcareassociated infections. Antimicrob Resist Infect Control 1:28. https://doi. org/10.1186/2047-2994-1-28 